\title{
Reversibility of tachycardia-induced cardiomyopathy after radiofrequency ablation of incessant supraventricular tachycardia in infants
}

\author{
Cristina Sanchez, Fernando Benito, Felipe Moreno
}

\begin{abstract}
Tachycardia-induced cardiomyopathy developed in a 3 month old infant with permanent junctional reciprocating tachycardia, which was incessant despite medical treatment. The patient underwent transcatheter radiofrequency ablation. There were no complications and 8 months after the procedure the patient was symptom free without medication.
\end{abstract}

(Br Heart f 1995;74:332-333)

Keywords: junctional reciprocating tachycardia; cardiomyopathy; radiofrequency ablation.

Permanent junctional reciprocating tachycardia (PJRT) is an uncommon form of incessant supraventricular tachycardia that occurs predominantly in infancy and childhood. Because PJRT is often resistant to medical treatment, such patients are at a high risk of developing tachycardia-induced cardiomyopathy. ${ }^{1}$ Lately radiofrequency transcatheter ablation has become established as an effective and safe treatment to eliminate accessory pathways in adults and children. ${ }^{2-4} \mathrm{We}$ describe successful radiofrequency ablation of PJRT in an infant with tachycardia-induced cardiomyopathy.

\section{Case report}

The patient, a 3 month old girl, was symptom free until she was 2 months old, when progressive feeding difficulties and tachypnoea were noted. The initial physical examination found signs and symptoms of heart failure with a heart rate of 230 beats $/ \mathrm{min}$. She weighed $4.5 \mathrm{~kg}$. The characteristics of the surface electrocardiogram were consistent with the diagnosis of PJRT: narrow-complex tachycardia with negative $P$ wave in leads II, III, and aVF and a long RP interval. Echocardiography showed left ventricular dilation and a shortening fraction of $20 \%$. The patient was treated for both heart failure and tachycardia with digoxin, diuretics, and amiodarone for 20 days. Because tachycardia remained incessant we decided to attempt radiofrequency ablation. Informed consent was obtained from her parents.
The ablation procedure was performed under general anaesthesia with intubation and mechanical ventilation. Three $5 \mathrm{~F}$ quadripolar electrode catheters were inserted percutaneously into the femoral and the right internal jugular veins and advanced to the high lateral right atrium, the right ventricular apex, the His bundle area, and the coronary sinus. We confirmed that the mechanism of the tachycardia, was a left posteroseptal pathway with long conduction time and decremental properties. A deflectable $5 \mathrm{~F}$ bipolar electrode catheter with a $3 \mathrm{~mm}$ tip (Mansfield/Webster) was positioned within the coronary sinus through the inferior vena
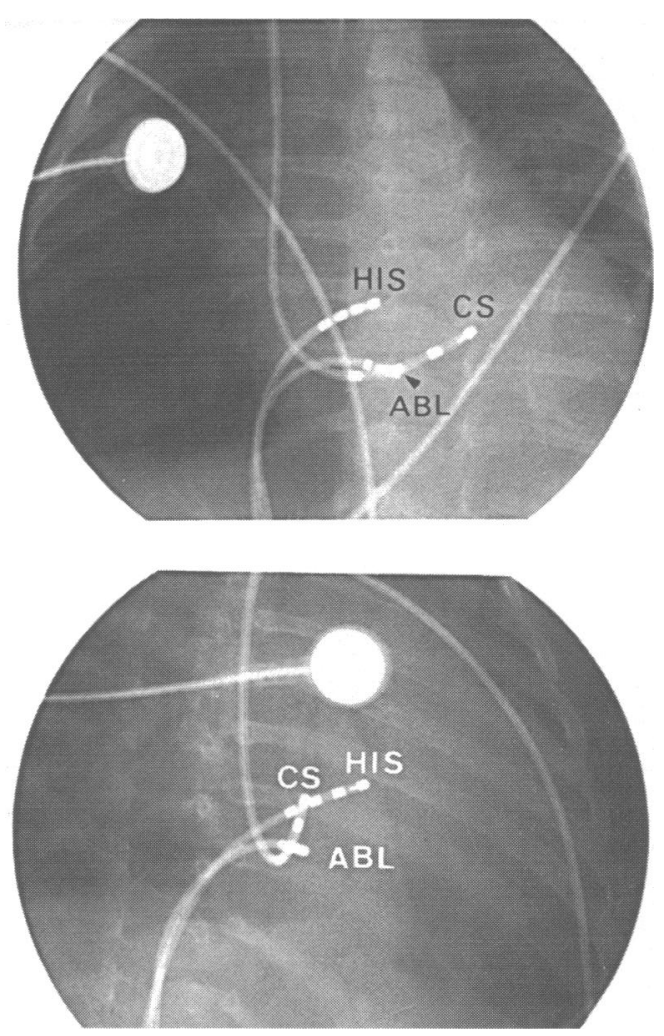

Figure 1 Chest radiographs showing catheter electrode positions during ablation of the left posteroseptal pathway. The ablating catheter is positioned within the coronary sinus close to the third electrode of the coronary sinus catheter. The upper panel shows the left anterior oblique projection and the bottom panel shows the right anterior oblique projection. ABL, ablation catheter; CS, coronary sinus catheter; His, His bundle catheter. 
Figure 2

Electrocardiograms

(ECGs) and intracavitary electrograms during supraventricular

tachycardia. From top to bottom: ECG leads II and

$V I$, His bundle electrogram (HBE), bipolar and unipolar electrograms from the mapping/ablation catheter $\left(M A P_{b i p}\right.$ and $M A P_{\text {uni }}$ ) and proximal, mid, and distal unipolar electrograms from the coronary sinus catheter $\left(C S_{4}, C S_{3}\right.$, and $\left.C S_{2}\right)$. Note that the retrograde atrial activation during supraventricular tachycardia recorded from the ablation catheter (dotted line) is earlier than the earliest retrograde atrial electrogram from the coronary sinus catheter $\left(C S_{3}\right)$. The probable accessory pathway potential precedes the atrial deflection (arrows).
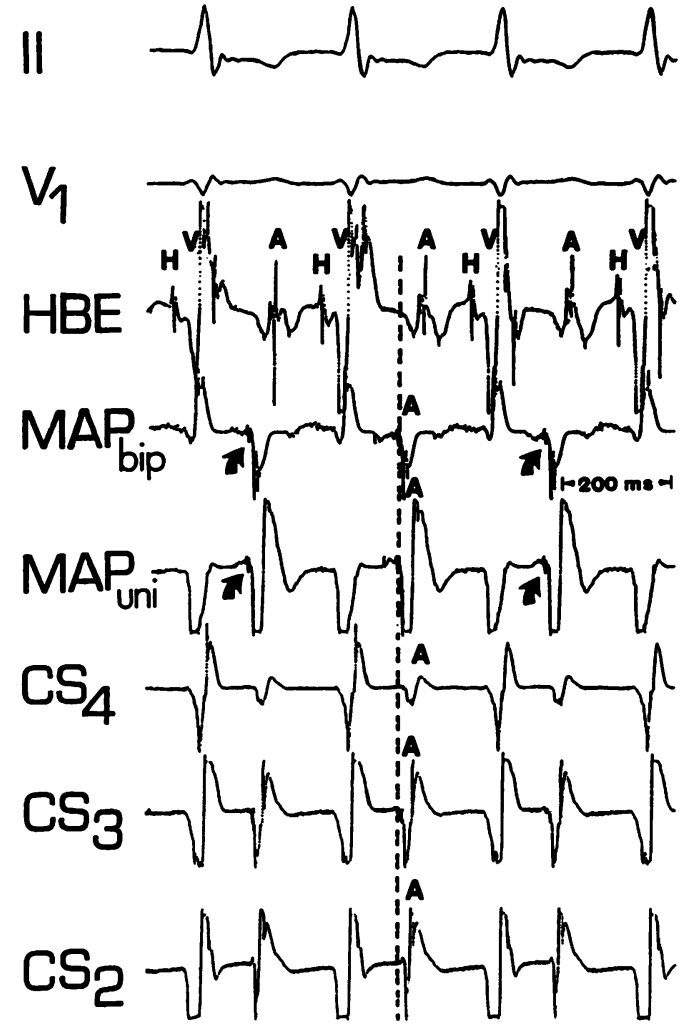

cava (figs $1 \& 2$ ). The radiofrequency current was set at $25 \mathrm{~W}$ for $60 \mathrm{~s}$. The tachycardia was terminated in the first second. The total procedure time was 3 hours with a total fluoroscopy time of 24 minutes. There were no complications. Subsequently ventricular function progressively improved. Echocardiography a week after ablation showed a shortening fraction of $30 \%$. Eight months after ablation she was symptom free without medication and the surface electrocardiogram showed sinus rhythm.

\section{Discussion}

The existence of tachycardia-induced cardiomyopathy caused by persistent abnormal high rates is well established. ${ }^{65}$ It is especially common in patients who have PJRT. Tachycardia can be asymptomatic for a long time before heart failure develops. ${ }^{1}$ Our patient, however, presented with tachycardiainduced cardiomyopathy. It is vital to make the correct diagnosis in these patients because ventricular dysfunction does not usually improve with conventional treatment. ${ }^{14}$ Von Hare et al reported successful ablation in a 2 month old infant with left ventricular dysfunction caused by PJRT. ${ }^{6}$ In their patient the ventricular accelerated rhythm that developed after ablation was treated with amiodarone. There were no complications in our patient. Our experience suggests that radiofrequency transcatheter ablation may be the best treatment in children with PJRT that does not respond to medical treatment. The presence of tachycardia-induced cardiomyopathy is an indication for radiofrequency ablation even in small infants.

1 Cruz FES, Cheriex EC, Smeets JLRM, Atié J, Peres AK, Penn OCKM, et al. Reversibility of tachycardia-induced cardiomyopathy after cure of incessant supraventricular tachycardia. F Am Coll Cardiol 1990;16:739-44.

2 Dick II M, O'Connor BK, Serwer GA, LeRoy S, Armstrong B. Use of radiofrequency current to ablate accessory connections in children. Circulation 1991;84: 2318-24.

3 Case CL, Gillette PC, Oslizlok PC, Knick BJ, Blair HL. radiofrequency catheter ablation of incessant, medically resistant supraventricular tachycardia in infants and small children. $₹ \mathrm{Am}$ Coll Cardiol 1992;20:1405-10.

4 Ticho BS, Saul JP, Hulse JE, De W, Lulu J, Walsh EP Variable location of accessory pathways associated with the permanent form of junctional reciprocating tachythe permanent form of junctional reciprocating tachycardia and confirmation with

5 Packer DL, Bardy GH, Worley SJ, Smith MS, Cobb FR, Coleman RE, et al. Tachycardia-induced cardiomyopathy; a reversible form of left ventricular dysfunction. Am $\mathcal{F}$ Cardiol 1986;57:563-57.

6 Van Hare GF, Witherell CL, Lesh MD. Follow-up of radiofrequency catheter ablation in children: results in 100 consecutive patients. $\mathcal{F} \mathrm{Am}$ Col Cardiol 1994;23: 1651-9. 